\title{
Induction systemic therapy followed by surgery for stages II-III non-small cell lung cancer: steady efforts
}

\author{
Ikuo Sekine, Yuka Aida, Hideo Suzuki \\ Department of Medical Oncology, Faculty of Medicine, University of Tsukuba, Tennodai 1-1-1, Tsukuba, Ibaraki 305-8575, Japan \\ Correspondence to: Ikuo Sekine. Professor, Department of Medical Oncology, Faculty of Medicine, University of Tsukuba, Tennodai 1-1-1, Tsukuba, \\ Ibaraki 305-8575, Japan. Email: isekine@md.tsukuba.ac.jp. \\ Provenance: This is an invited Editorial commissioned by Executive Editor-in-Chief Jianxing He (Director of the Thoracic Surgery Department, The \\ First Affiliated Hospital of Guangzhou Medical University, Guangzhou, China). \\ Comment on: Cascone T, Gold KA, Swisher SG, et al. Induction Cisplatin Docetaxel Followed by Surgery and Erlotinib in Non-Small Cell Lung \\ Cancer. Ann Thorac Surg 2018;105:418-24.
}

Submitted Aug 17, 2018. Accepted for publication Sep 12, 2018.

doi: $10.21037 /$ jtd.2018.09.91

View this article at: http://dx.doi.org/10.21037/jtd.2018.09.91

Lung cancer is the leading cause of mortality worldwide with 1.6 million deaths in 2012. The prognosis of lung cancer remains very poor (1). For most countries, the agestandardized 5-year survival rate ranges from $10 \%$ to $20 \%$ for most countries (2). Non-small cell lung cancer (NSCLC) accounts for about $85 \%$ of all lung malignant tumors. The first-line treatment for NSCLC is determined on the principles according to the TNM stage, which is the most reliable prognostic factor for this disease. Even patients with a localized tumor (stage I and stage II), as well as those with a resectable, locally advanced tumor (stage IIIA), have a poor prognosis when they are treated with surgery alone. In a Japanese study, the 5-year survival rate for pathological stages IB, IIA, IIB, and IIIA (categorized following the UICC-TNM staging system version 6) were $60-66 \%$, $59-60 \%, 42-47 \%$, and $28-33 \%$, respectively (3).

Since about $80 \%$ of recurrences are distant metastases (4) and many patients developed micrometastases at the time of surgery (5-7), chemotherapy has been added as a treatment postoperatively (adjuvant chemotherapy) or preoperatively (induction or neoadjuvant chemotherapy). The results of adjuvant chemotherapy in the controlled randomized trials are excellent; the first large-scale meta-analysis using individual data (lung adjuvant cisplatin evaluation, $n=4,584$ ) showed that $76 \%$ of patients received 3 to 4 cycles of chemotherapy, and the hazard ratio [95\% confidence interval (CI)] was $0.89(0.82-0.96)$, corresponding to a 5 -year absolute benefit of $5.4 \%$ from chemotherapy (8).
The second meta-analysis $(\mathrm{n}=8,447)$ and its follow-up data confirmed the results, with a hazard ratio $(95 \% \mathrm{CI})$ of $0.86(0.81-0.92)$ and a 5 -year absolute benefit of $4 \%$ $(9,10)$. However, these results from clinical trials should be interpreted with caution, because only the selected patients were included for the adjuvant clinical trials. For example, patients with pathological stage IIIB, incomplete resection, and unfavorable clinical course after surgery were all excluded from these trials. In other words, the advantages of the adjuvant approaches is the ability to select patients who are the good candidates for chemotherapy after surgery, and to predict the prognosis of patients based on an accurate pathological TNM stage.

Compared with adjuvant chemotherapy, induction chemotherapy has been less frequently tried because of the excellent advantages of adjuvant chemotherapy mentioned above. Induction chemotherapy; however, may have the following potential advantages: (I) increased compliance to chemotherapy; (II) better delivery of chemotherapy to the tumor tissue through an intact vasculature; (III) systemic treatment of occult microscopic metastases at the earliest possible time; (IV) assessment of tumor chemosensitivity in patients; and $(\mathrm{V})$ reduction of the primary tumor mass that can lead to an increase in resectability. The potential disadvantages of this approach are as follows: (I) delay in surgery; (II) decreased percentages of patients who receive a potentially curative surgery; (III) increased surgical mortality and morbidity; and (IV) less accurate staging (11-13). 
Table 1 Main randomized trials of neoadjuvant chemotherapy followed by surgery vs. surgery alone published in 2000

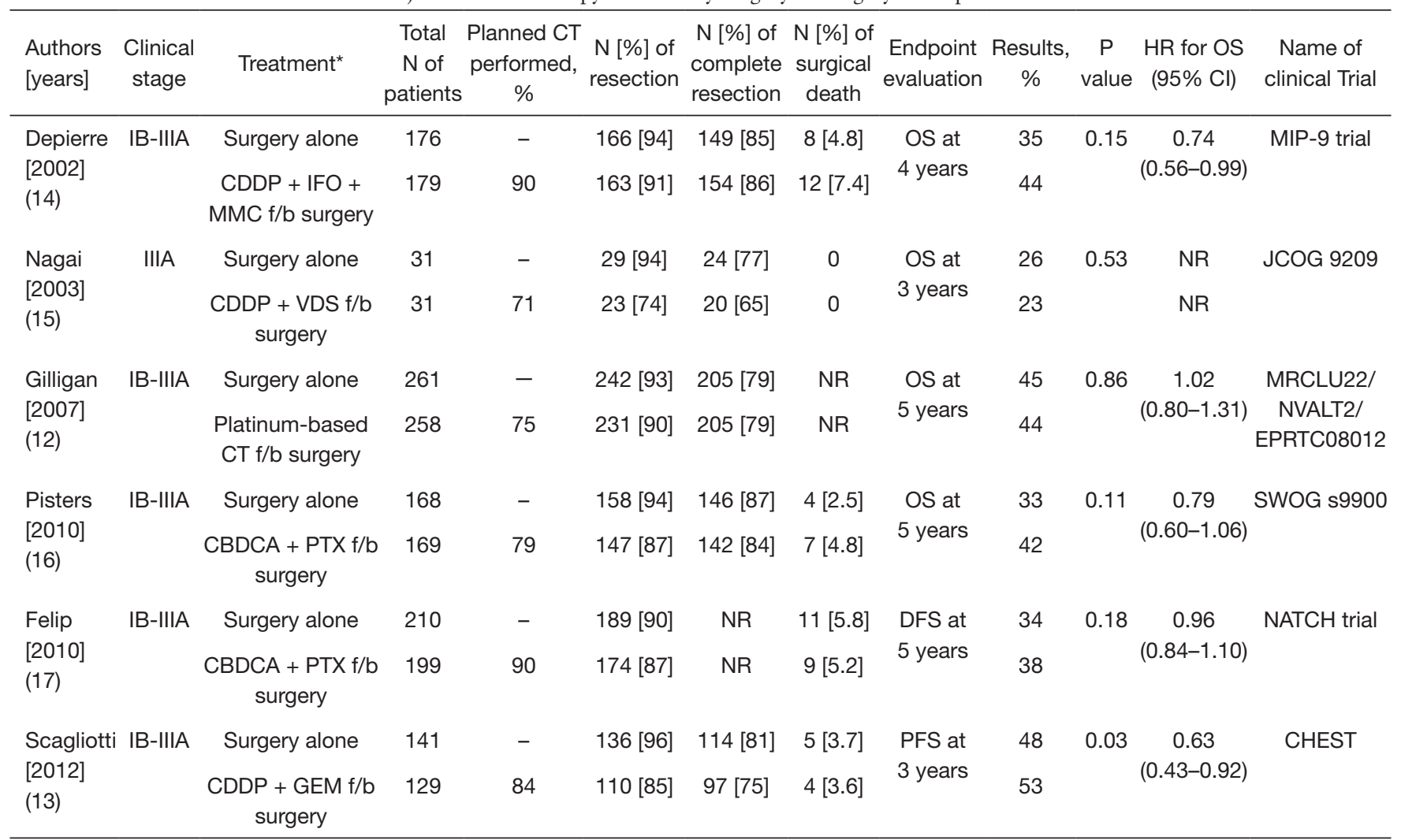

*Treatment: CBDCA, carboplatin; CDDP, cisplatin; GEM, gemcitabine; IFO, ifosfamide; MMC, mitomycin-C; PTX, paclitaxel; VDS, vindesine; CT, chemotherapy; DFS, disease-free survival; HR, hazard ratio; NR, not reported; OS, overall survival; PFS, progression-free survival.

Phase III trials of induction chemotherapy followed by surgery versus surgery alone fully published in 2000 are summarized in Table 1 (12-17). Although the compliance for induction chemotherapy remains high, either the percentage of resection or that of complete resection is not improved. A recent meta-analysis using individual patient data $(\mathrm{n}=2,385)$ showed a hazard ratio $(95 \% \mathrm{CI})$ of 0.87 (0.78-0.96), corresponding to a 5 -year absolute survival benefit of $5 \%$ (11). This figure is comparable to the results of adjuvant chemotherapy. A unique phase III trial (17) which compared using induction chemotherapy followed by surgery, surgery following adjuvant chemotherapy, and surgery alone, had revealed that as high as $90 \%$ of patients allocated to the induction chemotherapy arm received full cycles of chemotherapy. Whereas, only $61 \%$ of patients in the adjuvant chemotherapy arm received full cycles of chemotherapy. Tumor resection was observed in $87 \%$ of patients in the induction chemotherapy arm, $90 \%$ of patients in the adjuvant chemotherapy arm, and
$90 \%$ in the surgery alone arm. The 5-year disease-free survival, the primary endpoint of this study, was $38.3 \%$, $36.6 \%$, and $34.1 \%$ for the induction chemotherapy arm, adjuvant chemotherapy arm, and surgery alone arm, respectively, with no statistical significance. Thus, induction chemotherapy has potential advantages over surgery alone or even adjuvant chemotherapy following surgery, but this approach is still under investigation and should be performed in the clinical trial settings.

Cascone et al. reported a phase II trial of the three cycles of cisplatin $\left(80 \mathrm{mg} / \mathrm{m}^{2}\right)$ and docetaxel $\left(75 \mathrm{mg} / \mathrm{m}^{2}\right)$ induction chemotherapy followed by surgery and adjuvant erlotinib for 1 year in NSCLC patients with clinical stages IB to IIIA (18). Of the 47 eligible patients, 14 had stage IB, 13 had stage IIB, and 18 had stage IIIA. A total of 40 patients received the planned three cycles of chemotherapy, 4 patients received 2 cycles of chemotherapy and 3 patients received 1 cycle of chemotherapy. No cisplatin dose reduction was required in $87 \%$ of patients, and the planned 
docetaxel dose was maintained in $94 \%$ of patients. In spite of these good compliances to the chemotherapy, surgery was performed in only 37 (78.7\%) patients. Of them, 35 (74.5\%) underwent a complete resection. Adjuvant erlotinib was initiated in $21(45 \%)$ patients, and was completed for 1 year in $12(26 \%)$ patients. The 5 -year overall survival rate for patients with pretreatment stages I, II, and III were $51.9 \%, 55.5 \%$, and $21.1 \%$, respectively. The recurrence pattern was distant metastases in the majority $(85 \%)$ of cases. Induction cisplatin and docetaxel were well tolerated, but adjuvant erlotinib did not improve the outcomes when it was compared with the historical controls.

Complete tumor resection is the mainstay of treatment for resectable NSCLC without distant metastases. Thus, the percentages of patients who then proceeded to surgery and those who underwent a complete tumor resection are crucial in clinical trials of the induction chemotherapy. The actual resectability in phase II of the study was only $78.7 \%$, although the induction chemotherapy was well tolerated without the chemotherapy dose reduction in nearly $90 \%$ of patients. However, the reasons for not proceeding to surgery included an "excessive cardiopulmonary risk" in 4 of 10 patients who then gave up surgery as an option. This phrase, imprecise without a specific name of adverse events, implies that three cycles of cisplatin and docetaxel at full doses may be more exhausting than what was expected for the patients who were planning to undergo surgery, although this dose and schedule are tolerable for most of the patients who had been treated with chemotherapy alone. In this study, the complete resection rate of $74.5 \%$ is also disappointing. None of the patients had exhibited a complete radiological response, and only one patient achieved a pathological complete response in the primary tumor. Thus, it is clear that the efficacy of cisplatin and docetaxel, which is one of the most powerful platinum doubles for stage IV disease, is not sufficient for the use of induction chemotherapy.

Another investigational point of this phase II study is adjuvant erlotinib (18). However, the epidermal growth factor receptor (EGFR)-tyrosine kinase inhibitors were found to have a very limited role for the unselected patients who did not undergo an EGFR mutation analysis, and this was also demonstrated in adjuvant settings (19).

To date, the standard care of patients with clinical stages II-III NSCLC remains upfront surgery followed by a platinum-based adjuvant chemotherapy, according to the pathological findings in the resected tumor and the general condition of the patients after surgery. Then, is there no scope for induction drug therapy in these patients? A recent pilot study of two doses of nivolumab followed by surgery in 22 patients with a surgically resectable clinical stage IIIIA NSCLC showed that the treatment-related adverse events of any grade occurred in only $5(23 \%)$ patients, and grade 3-4 adverse events in 1 (4.5\%) patient. All 21 eligible patients who had underwent surgery, had no treatmentrelated delays and 20 (95\%) underwent complete resection. Radiographic evaluation of 21 patients showed 2 (9.5\%) partial responses and 18 (86\%) stable diseases. In the pathological evaluation of surgical specimens, results showed that $9(45 \%)$ of 20 patients had a major response and 2 (10\%) had complete disappearance of viable tumor cells (20). Thus, administration of induction immune checkpoint inhibitors before surgery can be feasible without delaying curative surgery and is highly effective. An experimental model using triple-negative breast cancer-bearing mice showed that paclitaxel was more effective when given after surgery than before surgery, whereas anti-PD-1 antibody was more effective in eradicating disseminated tumors when given before surgery than after surgery (21). These data points suggest that immunotherapy, including immune checkpoint inhibitors, may work better in the induction phase before surgery.

\section{Acknowledgements}

The author would like to thank Editage (www.editage.jp) for English language editing.

\section{Footnote}

Conflicts of Interest: Honoraria-Chugai Pharmaceutical Co., AstraZeneca K.K., Ono Pharmaceutical Co., Ltd, MSD K.K., and Nippon Boehringer Ingelheim Co., Ltd.

\section{References}

1. Ferlay J, Soerjomataram I, Dikshit R, et al. Cancer incidence and mortality worldwide: sources, methods and major patterns in GLOBOCAN 2012. Int J Cancer 2015;136:E359-86.

2. Allemani C, Matsuda T, Di Carlo V, et al. Global surveillance of trends in cancer survival 2000-14 (CONCORD-3): analysis of individual records for 37 513025 patients diagnosed with one of 18 cancers from 322 population-based registries in 71 countries. Lancet 2018;391:1023-75. 
3. Asamura H, Goya T, Koshiishi Y, et al. A Japanese Lung Cancer Registry study: prognosis of 13,010 resected lung cancers. J Thorac Oncol 2008;3:46-52.

4. Dziedzic DA, Rudzinski P, Langfort R, et al. Risk Factors for Local and Distant Recurrence After Surgical Treatment in Patients With Non-Small-Cell Lung Cancer. Clin Lung Cancer 2016;17:e157-67.

5. Pantel K, Izbicki JR, Angstwurm M, et al. Immunocytological detection of bone marrow micrometastasis in operable non-small cell lung cancer. Cancer Res 1993;53:1027-31.

6. Passlick B, Kubuschok B, Izbicki JR, et al. Isolated tumor cells in bone marrow predict reduced survival in nodenegative non-small cell lung cancer. Ann Thorac Surg 1999;68:2053-8.

7. Sienel W, Mecklenburg I, Dango S, et al. Detection of MAGE-A transcripts in bone marrow is an independent prognostic factor in operable non-small-cell lung cancer. Clin Cancer Res 2007;13:3840-7.

8. Pignon JP, Tribodet H, Scagliotti GV, et al. Lung adjuvant cisplatin evaluation: a pooled analysis by the LACE Collaborative Group. J Clin Oncol 2008;26:3552-9.

9. Arriagada R, Auperin A, Burdett S, et al. Adjuvant chemotherapy, with or without postoperative radiotherapy, in operable non-small-cell lung cancer: two meta-analyses of individual patient data. Lancet 2010;375:1267-77.

10. Burdett S, Pignon JP, Tierney J, et al. Adjuvant chemotherapy for resected early-stage non-small cell lung cancer. Cochrane Database Syst Rev 2015;CD011430.

11. NSCLC Meta-analysis Collaborative Group. Preoperative chemotherapy for non-small-cell lung cancer: a systematic review and meta-analysis of individual participant data. Lancet 2014;383:1561-71.

12. Gilligan D, Nicolson M, Smith I, et al. Preoperative chemotherapy in patients with resectable non-small cell lung cancer: results of the MRC LU22/NVALT 2/ EORTC 08012 multicentre randomised trial and update of systematic review. Lancet 2007;369:1929-37.

13. Scagliotti GV, Pastorino U, Vansteenkiste JF, et al. Randomized phase III study of surgery alone or surgery

Cite this article as: Sekine I, Aida Y, Suzuki H. Induction systemic therapy followed by surgery for stages II-III non-small cell lung cancer: steady efforts. J Thorac Dis 2018;10(Suppl 33):S3942-S3945. doi: 10.21037/jtd.2018.09.91 plus preoperative cisplatin and gemcitabine in stages IB to IIIA non-small-cell lung cancer. J Clin Oncol 2012;30:172-8.

14. Depierre A, Milleron B, Moro-Sibilot D, et al. Preoperative chemotherapy followed by surgery compared with primary surgery in resectable stage I (except T1N0), II, and IIIa non-small-cell lung cancer. J Clin Oncol 2002;20:247-53.

15. Nagai K, Tsuchiya R, Mori T, et al. A randomized trial comparing induction chemotherapy followed by surgery with surgery alone for patients with stage IIIA N2 nonsmall cell lung cancer (JCOG 9209). J Thorac Cardiovasc Surg 2003;125:254-60.

16. Pisters KM, Vallieres E, Crowley JJ, et al. Surgery with or without preoperative paclitaxel and carboplatin in earlystage non-small-cell lung cancer: Southwest Oncology Group Trial S9900, an intergroup, randomized, phase III trial. J Clin Oncol 2010;28:1843-9.

17. Felip E, Rosell R, Maestre JA, et al. Preoperative chemotherapy plus surgery versus surgery plus adjuvant chemotherapy versus surgery alone in early-stage nonsmall-cell lung cancer. J Clin Oncol 2010;28:3138-45.

18. Cascone T, Gold KA, Swisher SG, et al. Induction Cisplatin Docetaxel Followed by Surgery and Erlotinib in Non-Small Cell Lung Cancer. Ann Thorac Surg 2018;105:418-24.

19. Goss GD, O'Callaghan C, Lorimer I, et al. Gefitinib versus placebo in completely resected non-small-cell lung cancer: results of the NCIC CTG BR19 study. J Clin Oncol 2013;31:3320-6.

20. Forde PM, Chaft JE, Smith KN, et al. Neoadjuvant PD-1 Blockade in Resectable Lung Cancer. N Engl J Med 2018;378:1976-86.

21. Liu J, Blake SJ, Yong MC, et al. Improved Efficacy of Neoadjuvant Compared to Adjuvant Immunotherapy to Eradicate Metastatic Disease. Cancer Discov 2016;6:1382-99.

(English Language Editor: Jeremy Dean Chapnick, AME Publishing Company) 